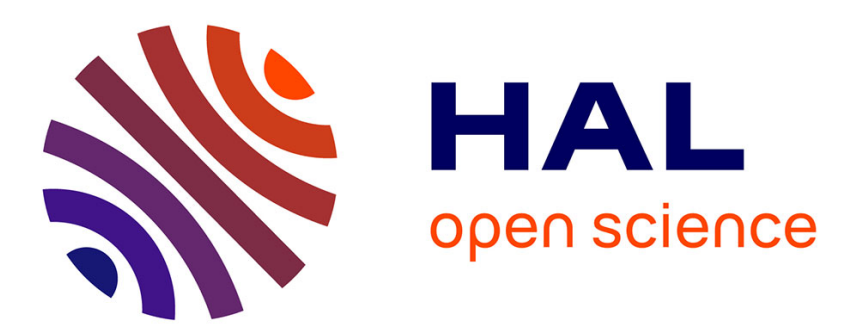

\title{
Utilisation et modélisation probabiliste des résultats d'inspection
}

\author{
Antoine Rouhan, Franck Schoefs
}

\section{To cite this version:}

Antoine Rouhan, Franck Schoefs. Utilisation et modélisation probabiliste des résultats d'inspection. Revue Française de Génie Civil , 2002, 6 (3), pp.505-514. 10.1080/12795119.2002.9692385 hal01007417

\section{HAL Id: hal-01007417 https://hal.science/hal-01007417}

Submitted on 8 Apr 2017

HAL is a multi-disciplinary open access archive for the deposit and dissemination of scientific research documents, whether they are published or not. The documents may come from teaching and research institutions in France or abroad, or from public or private research centers.
L'archive ouverte pluridisciplinaire HAL, est destinée au dépôt et à la diffusion de documents scientifiques de niveau recherche, publiés ou non, émanant des établissements d'enseignement et de recherche français ou étrangers, des laboratoires publics ou privés. 


\section{Utilisation et modélisation probabiliste des résultats d'inspection}

\section{Antoine Rouhan - Franck Schoefs}

Laboratoire de Génie Civil de Nantes Saint-Nazaire

Faculté des Sciences

2 rue la Houssinière

B.P. 92208, F-44322 Nantes cedex 03

\{Antoine.Rouhan, Franck.Schoefs\}@physique.univ-nantes.fr

RÉSUMÉ. Le suivi de grands ouvrages de Génie Civil passe par la mise en place de plans d'Inspection, Réparations, et Maintenance (IMR). De tels plans peuvent représenter des coûts dépassant la moitié du prix global de construction. Afin de réduire ces coûts, une optimisation est nécessaire. L'article se concentre sur les aspects liés aux inspections à base de contrôles non destructifs. A partir de la théorie de la détection et d'une analyse de coût simplifiée, on montre comment évaluer les surcoûts dus aux erreurs d'inspection, en soulignant l'importance des fausses détections (PFA). Enfin, à partir des courbes de performance des techniques d'inspection, il est possible d'en établir une hiérarchie. Le contexte du secteur offshore est pris comme exemple.

ABSTRACT. An operator of large civil engineering structures has to ensure that structural integrity is maintained to a sufficient level during in-service life, or during the total operating life if it is to be prolongated. This can be achieved by Inspection, Maintain and Repairs plans (IMR). Such plans are complex and can be expensive. This leads to the global optimization of these plans, and particularly inspection plans. In this context, we present original results on the use of inspections results in IMR plans. This approach is based on the decision and detection theory for inspection and include both the probability of false alarm and the probability of detection. It is shown how to use them. The effect of false alarm and miss detections on the global cost of inspections planning is highlighted through a general example. Endly it is shown how to rank inspections techniques on this basis.

MOTS-CLÉS : optimisation des inspections, structures existantes, probabilité de détection, probabilité de fausse alarme, contrôles non destructifs.

KEYWORDS: Inspections optimization, existing structures, probability of detection, probability of false alarm, non destructive testing. 


\section{Introduction}

Les ouvrages de génie civil existants doivent être surveillés durant toute la durée de la phase d'exploitation. Les objectifs sont la réparation ou le remplacement des composants suivant une stratégie donnée, l'inspection des zones structurelles sensibles de l'ouvrage et, enfin, d'assurer une maintenance régulière afin de garantir à l'ouvrage sécurité et fonctionnement. Ce sont les plans dits IMR pour Inspections, Maintenance et Réparations. Pour les grands ouvrages, il y a besoin d'optimiser ces plans, en termes de coûts et de performances. Un plan optimal serait d'inspecter au bon endroit au bon moment, avec les outils adaptés donnant des informations fiables, au prix le plus compétitif. Il en est de même pour la réparation et la maintenance. Depuis quelques années il y a une demande croissante pour de tels plans dans le domaine du génie civil et particulièrement pour l'estimation de la durée de vie résiduelle des structures arrivant en fin de vie. C'est le cas pour les ponts, barrages, centrales nucléaires et plates-formes offshore, qui ont été conçues pour une durée de vie de 20 à 50 ans, voire 100 ans pour les ouvrages exceptionnels, et dont la vie doit être prolongée pour des raisons économiques.

L'optimisation des plans IMR est typiquement un problème de décision. Dans ce papier, les aspects liés aux inspections sont mis en avant. Dans un premier temps, les inspections seront décrites d'un point de vue théorique. Il y est montré en particulier que le couple (PoD, PFA) (c'est-à-dire Probabilité de Détection, et Probabilité de Fausse Alarme) est nécessaire pour caractériser complètement les résultats d'inspections ainsi que les performances de l'appareil de mesure. Ensuite, dans un contexte de plan IMR, on introduit ce couple dans un schéma d'optimisation. Les effets des mauvaises performances d'inspections sont traduits en termes de coûts à travers un exemple, montrant les effets négatifs d'une PFA élevée. Le rôle central des fausses alarmes lors de l'utilisation des résultats d'inspection est souligné, car elles peuvent introduire de faux scénarios de ruine dont le coût des réparations inutiles peut être élevé.

\section{Stratégies de suivi des ouvrages en service}

Pour les grands ouvrages de génie civil, les actions de type IMR sont planifiées pour la durée de vie de la structure. Ceci signifie que l'on peut prendre en compte au moment de la conception :

- les dates et fréquences d'inspection, ainsi que les méthodes et techniques utilisées, dépendant de leurs performances et des capacités à être mises en œuvre in-situ,

- les dates et fréquences de maintenance,

- les réparation et/ou changement des composants si possible ainsi que les critères de décision associés.

Ceci permet une première évaluation du coût total du plan IMR. Pour les ouvrages déjà en service, le problème est spécifique, et l'on peut considérer deux cas : 


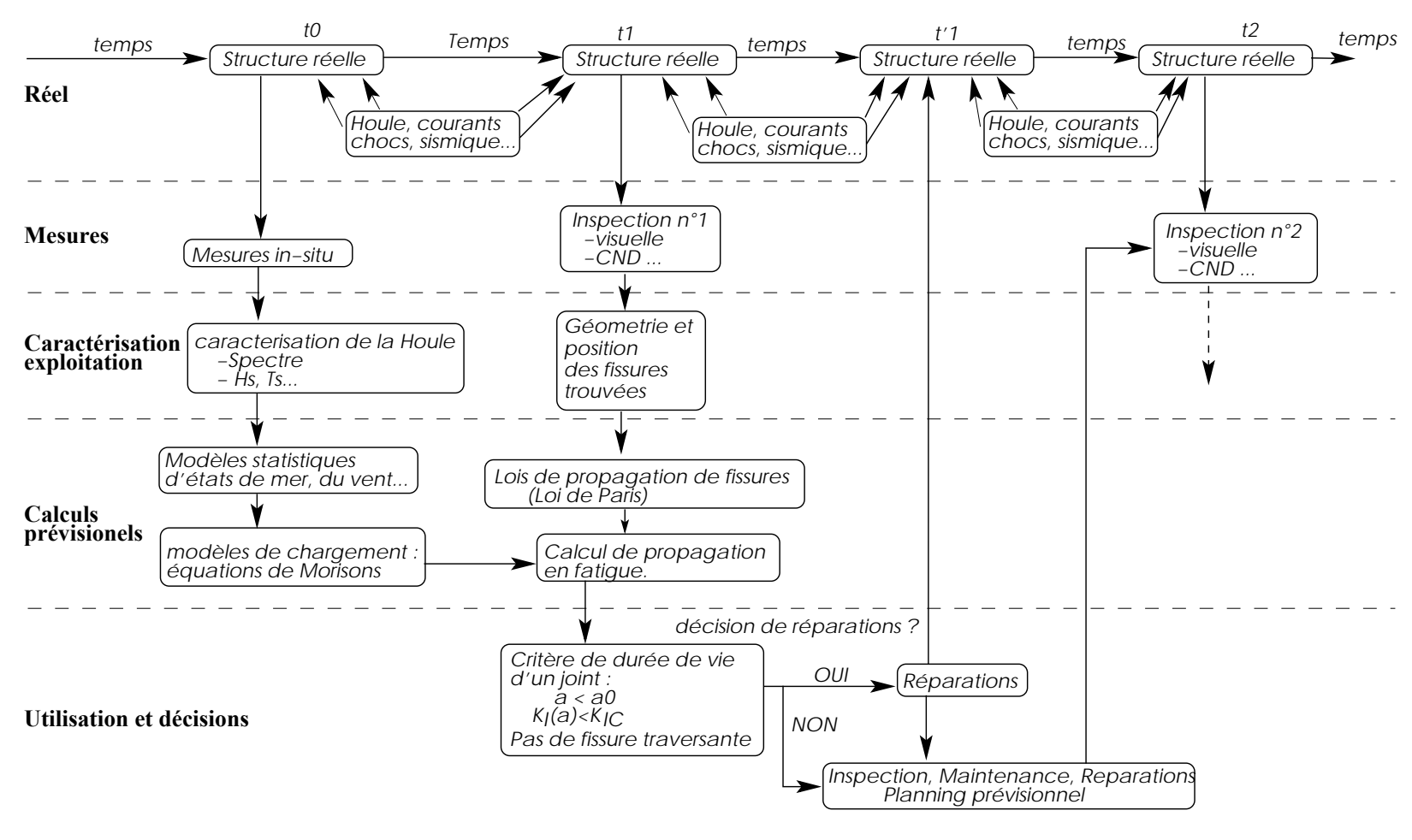

Figure 1. Exemple de plan IMR typique pour plates-formes offshore

- le cas où un grand nombre de points sensibles existe, et il devient alors nécessaire d'optimiser les zones d'inspection,

- le cas où, après inspection, des dommages non initialement prévus ont été découverts, et où le plan IMR initial doit être modifié, afin d'assurer la sécurité de l'ouvrage. Ceci signifie qu'il faut ré-estimer l'intégrité structurelle de l'ouvrage.

Dans un contexte de structure en service, la figure 1 présente un diagramme simplifié IMR ainsi que le schéma de décision associé pour la fatigue des plates-formes offshore. Un des dommages les plus importants pour de telles structures est la fatigue des nœuds tubulaires soudés. Les aspects liés à la maintenance ne sont pas représentés, étant considérés comment faisant partie des inspections.

\section{Les inspections par des techniques de contrôles non destructifs}

Les inspections sont une étape essentielle, car c'est le seul moyen pour avoir une vue au moins partielle de l'intégrité structurelle locale. Pour de grands ouvrages, une inspection optimale se fait dans la zone où les dommages structurels sont vraisemblablement importants, et avec une technique adaptée. Sur les structures existantes, les techniques de contrôle non destructives (CND) sont largement employées. Dans ce papier, nous porterons notre attention sur la détection de fissures, pathologie courante, aussi bien dans le béton que dans l'acier, et souvent représentative d'une perte d'intégrité locale significative. Tous les équipements CND ont des limitations et, dans des environnements complexes, leurs capacités sont différentes de celles données par 
les laboratoires ou les fabriquants. C'est le cas pour les inspections sous-marines de plates-formes offshore, les barrages, les centrales nucléaires et pour certaines parties structurelles des ponts, où l'accessibilité est réduite. Ceci conduit alors à des performances plus faibles que celles attendues.

\section{Performance des inspections}

Un challenge dans la stratégie des plans IMR est d'utiliser le maximum d'information disponible sur les performances des techniques CND afin d'optimiser leur utilisation. En général, les résultats d'inspection sont mis uniquement en rapport avec la probabilité de détection $(\mathrm{PoD})$, qui est la probabilité de détecter une fissure existante, avec une taille minimale de défaut détectable $a_{d}$ en dessous de laquelle on suppose qu'aucune détection n'est faite. Alors, la probabilité de détection est définie par :

$$
P o D(a)=P\left(a>a_{d}\right)
$$

où $a$ est la longueur de fissure. Dans un schéma probabiliste $a$ et $a_{d}$ sont des variables aléatoires, voir [MAD 87, GUO 92, GOY 94]. Dans la suite nous considérerons uniquement les détections, pas les tailles de fissure. Cependant, l'utilisation des PoD seules n'est pas satisfaisante : on doit en fait considérer une autre variable, qui est la probabilité de fausse alarme (en abrégé : PFA). Les fausses alarmes correspondent à la détection de fissures qui n'existent pas. Leur nature physique est le bruit et peut provenir de différentes sources : humaine, nature du phénomène à mesurer, conditions environnementales, etc. Il est nécessaire d'utiliser les PFA (voir [ROU 99]) car, pour les technologies sous-marines dans le domaine offshore par exemple, le fait de faire une fausse détection peut donner lieu à de faux scénarios de ruine, conduisant à des coûts logistiques de réparation inutiles. Du fait des conditions extrêmes dans lesquelles sont faites ces inspections, les fausses détections deviennent fréquentes et même de grandes fissures inexistantes peuvent être détectées. Dans la section suivante, à la fois PoD et PFA seront introduites d'un point de vue théorique.

\section{Introduction des inspections dans le processus de décision}

\subsection{Inspections : un peu de théorie}

Nous introduisons ici des développements fondamentaux de la théorie de l'inspection. En fait, faire une inspection est équivalent à prendre une décision. Pour illustrer ceci, prenons un exemple typique de détection de fissure. Supposons que l'on doive détecter une fissure existante dans un solide (ici, un nœud tubulaire offshore), avec un appareil CND donné. Après inspection, le résultat peut être : présence de fissure ou absence de fissure. En fait, ce résultat est une prise de décision sur l'état de la zone inspectée : fissurée ou non. On peut faire le même raisonnement dans le cas où il n'y pas de fissure présente sur la zone inspectée. L'ensemble est résumé figure 2. On voit 


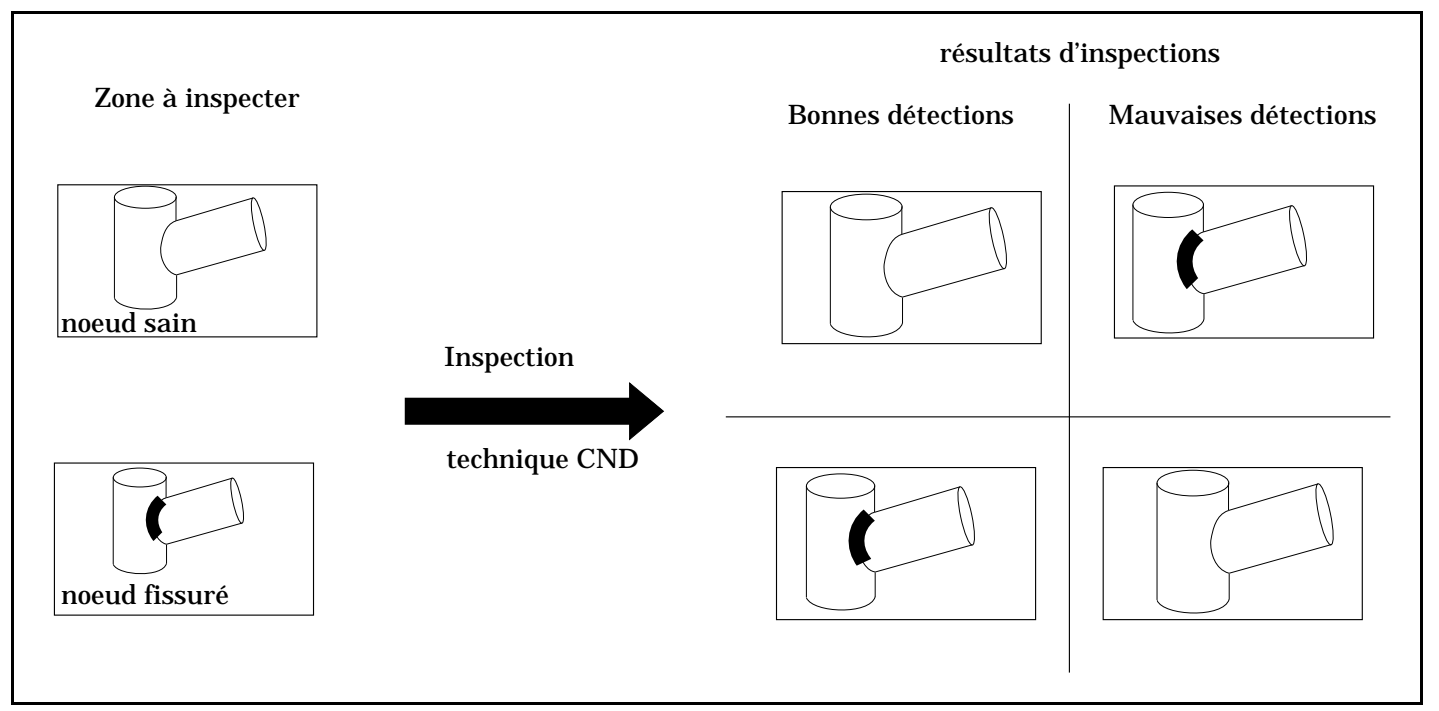

Figure 2. Illustration de la théorie de la détection

donc qu'il est nécessaire, dans un cadre IMR, de considérer les quatre événements suivants :

- $E_{1}$ : pas de fissure, sachant qu'il n'y a pas de fissure détectée,

$-\mathrm{E}_{2}$ : pas de fissure, sachant qu'une fissure est détectée : c'est une fausse alarme,

- $\mathrm{E}_{3}$ : présence de fissure, sachant qu'il n'y a pas de fissure détectée,

$-\mathrm{E}_{4}$ : présence d'une fissure, sachant qu'une fissure est détectée,

Dans la définition de ces événements, l'intérêt est porté sur la présence ou absence de fissure après inspection : le but est finalement de savoir si la fissure existe effectivement. Pour formaliser ceci, on introduit la théorie de la décision [ROU 99, CHA 90, ARQ 82]. D'un point de vue probabiliste, on considère une variable aléatoire binaire " présence de fissure » $X$, qui vaut zéro en cas d'absence de fissure, et un sinon. On note $d()$, la fonction d'inspection aléatoire (fonction de décision) qui vaut zéro si l'appareil CND décide de l'absence de fissure, un sinon. Alors, la probabilité de fausse alarme (PFA) et la probabilité de détection $(\mathrm{PoD})$ peuvent s'écrire :

$$
\begin{aligned}
\operatorname{PoD}(X) & =P(d(X)=1 \mid X=1) \\
\operatorname{PFA}(X) & =P(d(X)=1 \mid X=0)
\end{aligned}
$$

Ceci donne effectivement les définitions de la PoD et de la PFA [BAR 93, GOY 96] :

- la PoD est la probabilité de décider de la présence d'une fissure (détection), sachant que l'on a effectivement une fissure,

- la PFA est la probabilité de décider de la présence d'une fissure (détection), sachant que l'on n'a effectivement PAS de fissure. 
Notons que les résultats d'inspection peuvent être entièrement caractérisés par la donnée d'un couple (PoD,PFA). Notons $\gamma$ la probabilité de présence d'une fissure à l'endroit inspecté, on a alors :

$$
P(X=1)=\gamma, P(X=0)=1-\gamma
$$

La distribution de $\gamma$ en fonction de la taille de fissure existante et de la zone inspectée est rattachée à la distribution des tailles naturelles de fissures existantes ainsi qu'à leur distribution spatiale. Les probabilités de décision peuvent alors s'écrire :

$$
\begin{aligned}
& P(d(X)=1)=\operatorname{PFA}(X)(1-\gamma)+\operatorname{PoD}(X) \gamma \\
& P(d(X)=0)=(1-P F A(X))(1-\gamma)+(1-\operatorname{PoD}(X)) \gamma
\end{aligned}
$$

On a alors au final les probabilités suivantes :

$$
\begin{aligned}
& P\left(E_{1}\right)=P(X=0 \mid d(X)=0)=\frac{(1-P F A(X))(1-\gamma)}{(1-P o D(X)) \gamma+(1-P F A(X))(1-\gamma)} \\
& P\left(E_{2}\right)=P(X=0 \mid d(X)=1)=\frac{P F A(X)(1-\gamma)}{P o D(X) \gamma+P F A(X)(1-\gamma)} \\
& P\left(E_{3}\right)=P(X=1 \mid d(X)=0)=\frac{(1-P o D(X)) \gamma}{(1-P o D(X)) \gamma+(1-P F A(X))(1-\gamma)} \\
& P\left(E_{4}\right)=P(X=1 \mid d(X)=1)=\frac{P o D(X) \gamma}{P o D(X) \gamma+P F A(X)(1-\gamma)}
\end{aligned}
$$

On remarque que certains événements sont complémentaires. Par addition, on a :

$$
P\left(E_{1}\right)+P\left(E_{3}\right)=1, P\left(E_{2}\right)+P\left(E_{4}\right)=1
$$

Ceci signifie qu'un couple d'événements seul peut être considéré pour définir complètement l'inspection. Ceci est dû au fait que le couple (PoD,PFA) est suffisant pour représenter les capacités de détection d'un appareil CND.

\subsection{Analyse de coût}

L'optimisation des programmes d'inspection peut se faire en minimisant une fonctionnelle coût. Une analyse simplifiée consiste à calculer la fonctionnelle $E(C)$, définie par l'espérance du coût total :

$$
E(C)=\sum_{i} C\left(S_{i}\right) P\left(S_{i}\right)
$$

où :

- $C\left(S_{i}\right)$ est le coût associé au ième scénario,

- $P\left(S_{i}\right)$ est la probabilité que le ième scénario se produise. 
Par la suite, $\bar{C}_{i}$ est défini comme étant un surcoût résultant d'une mauvaise décision, conséquences incluses. Considérons les scénarios suivants relatifs au résultat d'une inspection donnant une bonne indication (bonne détection). On a, en utilisant les mêmes définitions que pour $E_{i}$ :

- $S_{1}$ : pas de fissure détectée, (et pas de fissure présente) le coût associé est : $C_{1}$,

$-S_{4}$ : fissure détectée, (et présence de fissure) le coût associé est $C_{4}$, y compris la réparation.

Considérons les scénarios duaux, donnant de fausses indications :

$-S_{2}$ : pas de fissure présente, mais on en détecte une, le coût final est $C_{2}=$ $C_{1}+\bar{C}_{4}$.

- $S_{3}$ : présence de fissure, mais pas de détection, le coût est $C_{3}=C_{1}+\bar{C}_{1}$.

Dans le cas du scénario $S_{2}$, et en cas de décision de réparation, le coût devient identique à celui du scénario $4\left(C_{2}=C_{4}\right)$, et il n'y pas de conséquences en termes de ruine structurelle. Cependant, ceci n'est pas optimal, car le surcoût $\bar{C}_{4}$ est élevé, à cause des réparations. En cas de non-réparation, la fausse alarme génère un faux scénario de ruine, ce qui peut modifier les chemins critiques de ruine dans les arbres de scénarios, augmentant artificiellement le coût : alors en général on a $C_{1}+\bar{C}_{4}>C_{4}$. Quant au scénario $S_{3}$, et en fonction de l'importance structurelle de la fissure, le surcoût $\bar{C}_{1}$ peut être soit très important, si l'on manque par exemple une fissure critique d'un point de vue structurel, ou faible si l'on manque une fissure peu importante. Maintenant, il est possible d'évaluer le coût total (équation [8]), à l'aide des résultats d'inspection. En cas de détection (décision de la présence d'une fissure), en utilisant [8] et [7] :

$$
E(C)=\sum_{2,4} C\left(S_{i}\right) P\left(S_{i}\right)=C_{4} P\left(E_{4}\right)+\left(C_{1}+\bar{C}_{4}\right)\left(1-P\left(E_{4}\right)\right)
$$

En cas de non-détection (décision d'absence de fissure), et en utilisant [8] et [7] :

$$
E(C)=\sum_{1,3} C\left(S_{i}\right) P\left(S_{i}\right)=C_{1} P\left(E_{1}\right)+\left(C_{1}+\bar{C}_{1}\right)\left(1-P\left(E_{1}\right)\right)
$$

Les deux équations [9] et [10] comportent deux termes, l'un associé avec la bonne détection, l'autre avec la mauvaise détection. Elles sont dans un certain sens complémentaires, et les deux sont fonction des PoD, PFA et $\gamma$. Afin de mieux comprendre comment ces fonctions coûts varient en fonction des performances de détection des appareils CND, on étudie le comportement de ces fonctions.

\subsection{Illustration}

Nous étudions maintenant l'effet des surcoûts dus à de mauvaises détections, sur le coût total estimé. Dans les équations [9] et [10], l'espérance du surcoût est respectivement $\left(C_{1}+\bar{C}_{4}\right)\left(1-P\left(E_{4}\right)\right)$ et $\left(C_{1}+\bar{C}_{1}\right)\left(1-P\left(E_{1}\right)\right)$. En traçant les probabilités 

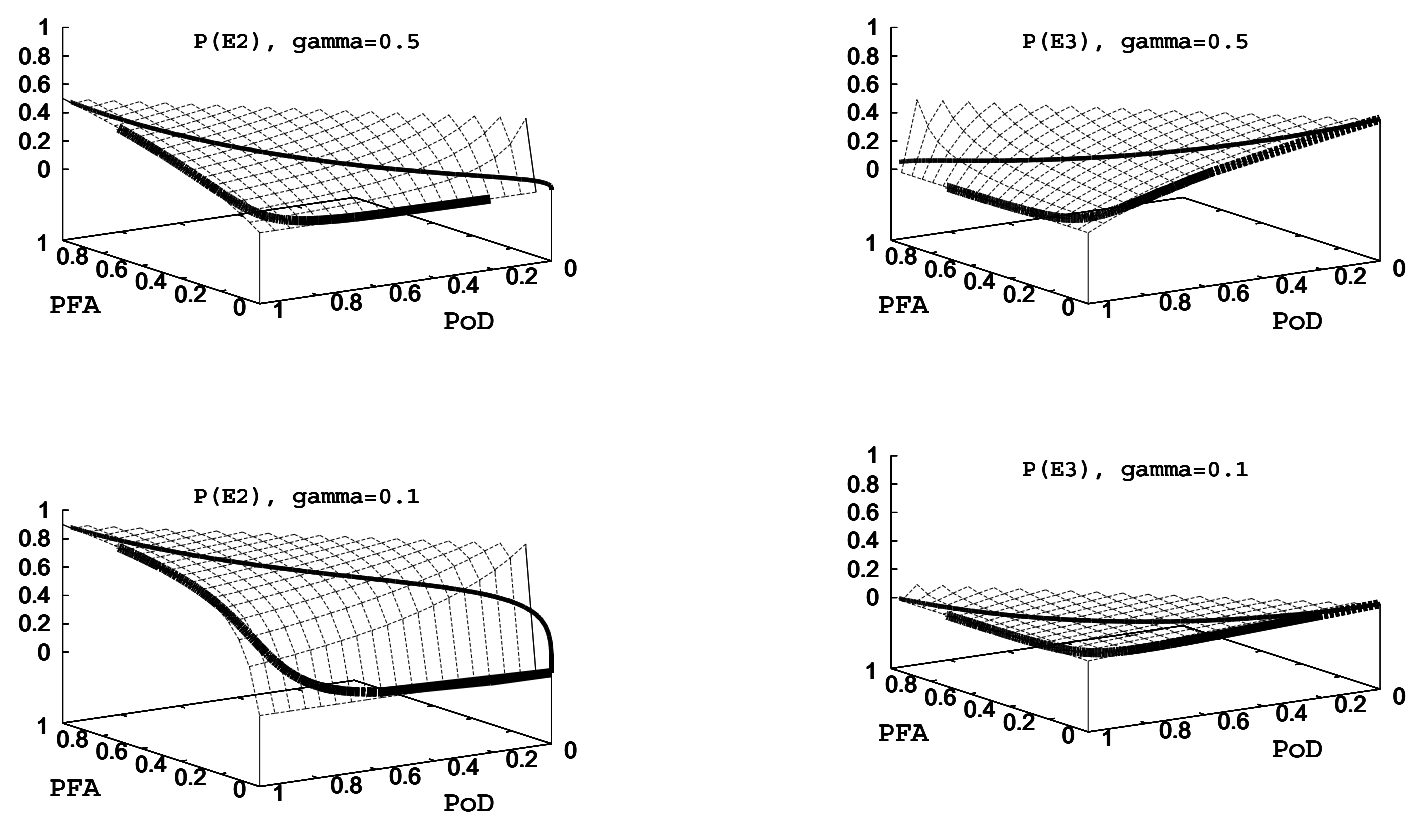

Figure 3. $P\left(E_{2}\right)$ pour $\gamma=(0.5,0.1)$

Figure 4. $P\left(E_{3}\right)$ pour $\gamma=(0.5,0.1)$

d'occurrence des faux scénarios $\left(1-P\left(E_{4}\right)\right)=P\left(E_{2}\right)$ et $\left(1-P\left(E_{1}\right)\right)=P\left(E_{3}\right)$, en fonction de PoD et PFA pour différentes valeurs de $\gamma$, on peut voir l'influence de ces paramètres sur les surcoûts. La figure 3 montre la surface $P\left(E_{2}\right)$, et la figure 4 la surface $P\left(E_{3}\right)$, définie sur le plan (PoD,PFA), pour deux valeurs de $\gamma$ :

$-\gamma=0.1$ qui représente une probabilité faible de présence de fissure. Ceci représente typiquement les moins fréquentes;

$-\gamma=0.5$ ce qui est typique des tailles de fissures couramment rencontrées.

Ces valeurs de $\gamma$ peuvent être déduites des densités de probabilité des tailles naturelles de fissures : [CIO 86] suppose une densité de probabilité exponentielle pour les tailles de fissures. On interprète alors les figures comme suit :

- pour $P\left(E_{2}\right)$, qui est associé avec les fausses alarmes (figure 3 ) on remarque des effets importants sur le surcoût en cas de fissures peu fréquentes, notamment si la PFA devient significative : $P\left(E_{2}\right)$ atteint 1 pour une grande partie des points du plan $(P o D, P F A)$. Il en résulte que le surcoût a une très grande probabilité de se produire. C'est pourquoi la PFA joue un rôle aussi important sur les plans IMR, pour les fissures peu fréquentes (que l'on peu associer aux fissures de grande taille);

- pour $P\left(E_{3}\right)$, qui est associé aux non détections, on a (figure 4) un effet très marqué du surcoût en cas de fissures courantes. Cependant, le coût peut être élevé en cas de propagation de petits défauts et sans inspection planifiée ni suivi de ces fissures (coût indirect).

Notons que les rôles de la PoD et de la PFA sont inversés pour les deux cas, pour une probabilité de présence de fissure $\gamma$ inversée. 


\subsection{Choix optimal d'une technique}

Cette analyse de coût nous permet de mettre en place un critère de classification des méthodes d'inspection. Leurs performances sont généralement traduites sous forme de courbes ROC (pour Caractéristiques Opérationnelles du Récepteur). Il s'agit de tracer $P o D(X)$ en fonction de $P F A(X)$, probabilités d'événements non complémentaires. On obtient alors la courbe ROC, qui est une caractéristique essentielle de la technique d'inspection [ARQ 82, ROU 01]. Le point idéal est le point où la PoD vaut 1 et la PFA vaut 0 . On a superposé sur les surfaces précédentes deux courbes ROC, représentant deux méthodes d'inspection, l'une performante en gras (très anguleuse, elle passe proche du point $(1,0))$, l'autre peu performante. En sachant qu'une inspection correctement faite par un opérateur qualifié situe la portion utile de la courbe ROC dans la zone la plus proche du point $(1,0)$, on peut facilement analyser les surcoûts dus à de mauvaises indications, et classer les techniques d'inspection en fonction du surcoût qu'elles génèrent. En se fixant ensuite un critère de coût maximal, on peut choisir de façon optimale quelle technique d'inspection choisir. Sur les graphiques, il apparaît clairement que la méthode d'inspection la plus performante minimise les surcoûts d'erreur d'inspection.

\section{Conclusions}

A partir d'une définition théorique des caractéristiques des méthodes de contrôles non destructifs, nous avons montré comment inclure les résultats des inspections dans un schéma probabiliste de type IMR. A l'aide d'une analyse de coût simplifiée, on a montré comment les probabilités de détection et de fausse alarme interviennent dans les aspects décisionnels, en soulignant l'importance des fausses alarmes en termes de surcoût. En générant de faux scénarios de ruine, les fausses alarmes modifient la prospection des arbres de ruine de la structure étudiée. On doit donc faire une analyse très critique de ces derniers, au vu des résultats d'inspections. Enfin, à partir de cette analyse de coût, et des performances intrinsèques des techniques CND, on a établi un critère permettant de les hiérarchiser. Ceci doit permettre à terme la mise au point d'un outil d'aide à la décision visant à optimiser globalement les plans d'inspection ainsi que les techniques mises en œuvre.

\section{Bibliographie}

[ARQ 82] ARquĖs P.Y., Décisions en traitement du signal, Éditions Masson, Paris, 1982, ISBN 2-2257-6758-0.

[BAR 93] Barnouin B., Lemoine L., Dover W.D., Rudlin J., FAbBri S., Rebourcet G., TOPP D., KARE R., SANGOUARD D., « Underwater inspection reliability trials for offshore structures », ASME N. Y., Ed., Proceeding of the 12th International Conference on Offshore Mechanics and Arctic Engineering, vol. 3B, 1993, p. 883-890. 
[CHA 90] CHARABit M., Éléments de théorie du signal : les signaux aléatoires, Éditions Ellipses, Paris, 1990.

[CIO 86] CIOCLOV D.D., "A model for fracture risk analysis », Revue roumaine de sciences techniques. Série de mécanique appliquée, vol. 6, 1986, p. 613-622, Fascicule 31.

[GOY 94] Goyet J., Paygnard J.C., Maroini A., Faber M.H., «Optimal inspection and repair planning : case studies using IMREL software », ASME N. Y., Ed., Proceeding of the 13th International Conference on Offshore Mechanics and Arctic Engineering, vol. 2, février 1994.

[GOY 96] GOYET J., Evaluation des apports de la technique d'inspection FMD, ATA 580 A OPI. Rapport interne Bureau Veritas, 1996.

[GUO 92] GUOYANG JiAO, « Reliability analysis of crack growth with inspection planning », ASME N. Y., Ed., Proceeding of the 11th International Conference on Offshore Mechanics and Arctic Engineering, vol. 3, juin 1992.

[MAD 87] Madsen H.O., SkJong R.K, TAllin A.G., KiRKemo F., « Probabilistic fatigue crack growth analysis of offshore structures, with reliability updating through inspection », Marine Structure Reliability Symposium, octobre 1987.

[ROU 99] Rouhan A., SchOefs F., LABEYRIE J., « Approche probabiliste du contrôle non destructif des structures pétrolières pour l'évaluation de leur intégrité structurelle », INSTRUC 4, Prévision et estimation de la durée de vie des structures mécaniques, vol. 1, novembre 1999.

[ROU 01] ROUHAN A., Étude sur les probabilités de détection des contrôles non destructifs, rapport, mai 2001, IFREMER, Rapport d'étude de contrat universitaire $n^{0} 002021316$ TMSI/RED/MS. 